\title{
ON THE STRUCTURE OF TAME NEAR-RINGS
}

\author{
H. HEATHERLY and G. PILZ
}

(Received 21 July 1989)

Communicated by B. J. Gardner

\begin{abstract}
Tame near-rings form an important class of near-rings. They have the common feature that all $N$-subgroups in a faithful $N$-group are ideals. Tame near-rings can be very close to and very far from rings. Most of the important classes of distributively generated near-rings and all 2-semisimple near-rings are examples of tame near-rings.
\end{abstract}

1980 Mathematics subject classification (Amer. Math. Soc.) (1985 Revision): 16 A 76.

If a near-ring $N$ is tame on some $N$-group $T$ then $T$ is of type 2 if and only if it is of type 0 . Hence it might make sense to ask if for tame near-rings these types 0 and 2 coincide on all $N$-groups. The answer is "yes", if a quite general condition is fulfilled, but we give a counterexample in the other case.

Definitions. Let $N$ be a right zero-symmetric near-ring, and let $T$ be an $N$-group. Then $T$ is said to be tame if every $N$-subgroup of $T$ is an ideal of $T$. If, moreover, $T$ is faithful then $N$ is said to be tame on $T$.

For more on tame near-rings, see the papers cited in the references. Now we are going to study the behaviour of a tame near-ring on some (other) $\mathrm{N}$ group $\Gamma$. For this, we need a slight generalization of a result of $G$. Betsch $[1,3.4(\mathrm{i})]$.

LEMMA. Let $N$ be a zero-symmetric near-ring, $\Gamma$ an $N$-group with $\Gamma=$ $N \gamma_{0}$ for some $\gamma_{0}$ in $\Gamma$, and $L_{1}, L_{2}$ be left ideals of $N$ such that $L_{1}+$ $\left(0: \gamma_{0}\right)=L_{2}+\left(0: \gamma_{0}\right)=N$, but $L_{1} \cap L_{2} \subseteq\left(0: \gamma_{0}\right)$. Then $N /(0: \Gamma)$ is a ring.

(C) 1991 Australian Mathematical Society 0263-6115/91 \$A2.00+0.00 
This follows easily from $[1,3.14(\mathrm{a})$ and $3.4(\mathrm{i})]$, applied to $\Gamma$ as an $N /(0: \Gamma)$-group.

Defintrion. Let $T$ and $\Gamma$ be $N$-groups. Then $T$ is said to be subversive to $\gamma_{0}$ in $\Gamma$ if $N \gamma_{0}=\Gamma$ and there is a family $\left(\tau_{\alpha}\right)_{\alpha \leq \lambda}$ in $T$ such that $\lambda$ is a limit ordinal and $\bigcap_{\alpha \leq \beta}\left(0: \tau_{\alpha}\right) \nsubseteq\left(0: \gamma_{0}\right)$ for all $\bar{\beta}<\lambda$, but $\bigcap_{\alpha \leq \lambda}\left(0: \tau_{\alpha}\right)$ $\subseteq\left(0: \gamma_{0}\right)$.

Since all $\left(0: \tau_{\alpha}\right)$ (and their intersections) are left ideals in $N$, we get

Proposition. If $N$ has the descending chain condition on left ideals then no $N$-group can be subversive.

TheOREM. Let $N$ be a zero-symmetric near-ring, $T$ be a tame $N$-group and let $\Gamma=N \gamma_{0}$ be of type 0 . If $(0: T) \subseteq\left(0: \gamma_{0}\right)$ and if $T$ is not subversive to $\gamma_{0}$ then $\Gamma$ is of type 2 .

Proof. Case (i): $\left(0: \tau_{0}\right) \subseteq\left(0: \gamma_{0}\right)$ holds for some $\tau_{0} \in T^{*}=T-\{0\}$. Then $h: N \tau_{0} \rightarrow \Gamma=N \gamma_{0}, n \tau_{0} \rightarrow n \gamma_{0}$, is a well-defined $N$-epimorphism. Also, $N \tau_{0}$ is tame. By [1,9.171], $N \tau_{0} / \operatorname{Ker} h$ (which is $N$-isomorphic to $\Gamma$ ) is tame. Hence $\Gamma$ is tame and thus of type 2 .

Case (ii): not (i). Then $(0: \tau) \nsubseteq\left(0: \gamma_{0}\right)$ holds for all $\tau \in T^{*}$.

Case (a): there is no $\Sigma \subset T^{*}$ such that $(0: \Sigma) \subseteq\left(0: \gamma_{0}\right)$,

$$
L_{1}:=(0: T-\{\tau\}) \nsubseteq\left(0: \gamma_{0}\right), \quad L_{2}:=(0: \tau) \nsubseteq\left(0: \gamma_{0}\right),
$$

but

$$
L_{1} \cap L_{2}=(0: T) \subseteq\left(0: \gamma_{0}\right) \quad \text { for each } \tau \in T^{*} .
$$

Since $\Gamma$ is of type 0 and $N /\left(0: \gamma_{0}\right) \cong_{N} \Gamma,\left(0: \gamma_{0}\right)$ is a maximal left ideal in $N$. Hence $L_{i}+\left(0: \gamma_{0}\right)=N$ for $i=1,2$. By the lemma, $N /(0: \Gamma)$ is a ring.

Case (b): there exists some $\Sigma \subset T^{*}$ with $(0: \Sigma) \subseteq\left(0: \gamma_{0}\right)$. Let $\Sigma=$ $\left\{\sigma_{\alpha} \mid \alpha \in A\right\}$ and let $A$ be well-ordered. Hence $\Sigma=\left\{\sigma_{0}, \sigma_{1}, \ldots\right\}$. Let $\omega$ be the ordinal of $A$. Take some $\tau \in T^{*}-\Sigma$ and add $\tau$ to $\Sigma$ as $\tau=\sigma_{\omega}$ (=last element of $\bar{\Sigma}:=\Sigma \cup\{\tau\})$. Then again $(0: \bar{\Sigma}) \subseteq\left(0: \gamma_{0}\right)$ and $\bar{\Sigma}=\left\{\sigma_{\alpha} \mid \alpha \leq \omega\right\}$. Take $B:=\left\{\alpha \in A \mid \bigcap_{i \leq \alpha}\left(0: \sigma_{i}\right) \subseteq\left(0: \gamma_{0}\right)\right\}$. Since $\omega \in B, B \neq \varnothing$ and hence $B$ contains a smallest element $\beta_{0}$. Take $L_{1}:=\bigcap_{\alpha<\beta_{0}}\left(0: \sigma_{\alpha}\right)$ and $L_{2}:=$ $\left(0: \sigma_{\beta_{0}}\right) \nsubseteq\left(0: \gamma_{0}\right)$. If $\beta_{0}$ is not a limit ordinal then $L_{1}=\bigcap_{\alpha \leq \beta_{0}-1}\left(0: \sigma_{\alpha}\right)$ $\nsubseteq\left(0: \gamma_{0}\right)$ and we might proceed as in (a) to show that $N /(0: \Gamma)$ is a ring. If $\beta_{0}$ is a limit ordinal, however, the family $\left(\sigma_{\alpha}\right)_{\alpha \leq \beta_{0}}$ is subversive to $\gamma_{0}$, a contradiction.

Hence in both cases (a) and (b), we know that $N /(0: \Gamma)$ must be a ring. Let $\Delta$ be an $N$-subgroup of $\Gamma$. We want to show that $\Delta$ is an ideal of $\Gamma$. For this, take $\delta=d \gamma_{0} \in \Delta, \gamma=n \gamma_{0} \in \Gamma$ and $A:=(0: \Gamma)$. 
(1) $(N / A,+)$ is abelian, so $n+d-n \equiv d(\bmod A)$. Thus

$$
\begin{aligned}
\gamma+\delta-\gamma & =n \gamma_{0}+d \gamma_{0}-n \gamma_{0} \\
& \left.=(n+d-n) \gamma_{0}=d \gamma_{0}+a \gamma_{0} \quad \text { (for some } a \in A\right) \\
& =d \gamma_{0}=\delta .
\end{aligned}
$$

Hence $\Gamma$ is abelian and $\Delta$ is normal.

If $n^{\prime} \in N$ then a similar argument shows that

$$
n^{\prime}(\gamma+\delta)-n \gamma=\left(n^{\prime}(d+n)-n^{\prime} n\right) \gamma_{0}=n^{\prime} d \gamma_{0}=n^{\prime} \delta \in \Delta .
$$

Hence $\Delta$ is an ideal in $\Gamma$ and hence trivial.

This shows that $\Gamma$ is of type 2 .

The subversity-condition in the theorem cannot be omitted.

EXAMPLE. Let $N$ be the near-ring of all analytic functions from $\mathbb{C}$ into $\mathbb{C}$ which have an (everywhere) convergent power series expansion at 0 with only real coefficients. Take $\Gamma=\mathbb{C}$ and $\Delta=\mathbb{R}$.

(i) $N$ can easily be seen to be 2-primitive on $\Delta$ (1 can serve as a generator; if $E$ would be a non-trivial $N$-subgroup, take $\varepsilon \in E^{*}, \delta \notin E$, $n=\delta z / \varepsilon$, then $n(\varepsilon)=\delta \notin E)$. Also, if $n$ is zero on $\Delta, n=0$.

(ii) Therefore, $N$ is tame on $\Delta$, too.

(iii) $\Gamma$ is an $N$-group of type 0 : as $\gamma_{0}$ we might take $i=\sqrt{-1}$, since if $a+b i \in \mathbb{C}$ and $n=b z-a z^{2}$ then $n(i)=a+b i$. If $\Theta$ is an ideal of $\Gamma$ then all $n(\gamma+\theta)-n(\gamma)$ must be contained in $\Theta$ for $\gamma \in \Gamma$ and $\theta \in \boldsymbol{\theta}$. Take $n=e^{z}$. Then

$$
n(\gamma+\theta)-n(\gamma)=e^{\gamma+\theta}-e^{\gamma}=e^{\gamma}\left(e^{\theta}-1\right) \in \mathbf{\theta} .
$$

If $\theta \neq 0$, it is always possible to get $e^{\gamma}\left(e^{\theta}-1\right)$ to be any value in $\mathbb{C}$. Hence $\theta$ must be trivial.

(iv) $N$ is even 0-primitive on $\Gamma$, but $\Gamma$ is not of type 2 , since it has $\Delta$ as a non-trivial $N$-subgroup.

(v) $T=\Delta$ is subversive to $\gamma_{0}=i$. To see this, take $\tau_{k}=k^{-1}(k \in \mathbb{N})$. Each $\bigcap_{k=1}^{m}\left(0: \tau_{k}\right) \nsubseteq(0: i)$, since, for example, $n=\prod_{k=1}^{m}\left(z-\tau_{k}\right)$ belongs to $\bigcap_{k=1}^{m}\left(0: \tau_{k}\right)$, but not to $(0: i)$. But $\bigcap_{k=1}^{\infty}\left(0: \tau_{k}\right)=0$, since any $n$ in this intersection has a non-isolated pole, something an analytic function cannot have.

(vi) $\left(0: \gamma_{0}\right)=(0: i) \neq 0$, since, for example, $n=z^{2}+1 \in(0: i)$.

COROLlary. Let $N$ be zero-symmetric and tame on $T$. If $T$ is not subversive to any $\gamma_{0}$ in some $N$-group $\Gamma$ then $\Gamma$ is of type 0 if and only if it is of type 2. 
COROLlary. Let $N$ be zero-symmetric, tame, and with the descending chain condition on left ideals. Then any $N$-group of type 0 is of type 2 , the $J_{0}$ - and the $J_{2}$-radicals coincide, and $J_{2}(N)$ is nilpotent.

Compare this corollary to $[1,9.188]$. Some interesting problems remain open.

Open problems. (i) Find a tame near-ring such that its $J_{0}$ - and $J_{2}$-radicals do not coincide.

(ii) If $I$ is an ideal of $N$, such that $I$ and $N / I$ are tame, is $N$ then necessarily tame, too?

\section{Acknowledgement}

This work was done while the second author was a Visiting Professor at the Department of Mathematics at the University of Southwestern Louisiana. He expresses his gratitude for the hospitality he received there, especially from Professor Blumberg.

\section{References}

[1] Günter Pilz, Near-rings, (North-Holland, Amsterdam, 1983, 2nd ed.).

[2] Stuart D. Scott, Tame theory, (Amo Publ. Co., Univ. Auckland, New Zealand).

University of Southwest Louisiana

Lafayette, Louisiana 70504

U.S.A.
Institut für Mathematik Universität Linz A-4040 Linz Austria 